\title{
Telerreabilitação em grupo para pacientes com dor lombar crônica: Estudo longitudinal retrospectivo
}

\author{
Group telerehabilitation for patients with chronic low back pain: A longitudinal retrospective study \\ Telerehabilitación en grupo para pacientes con dolor lumbar crónico: Estúdio retrospectivo
} longitudinal

\section{Resumo}

Objetivo: Avaliar os efeitos da telerreabilitação em grupo na dor e incapacidade de pacientes com dor lombar crônica. Metodologia: Trata-se de um estudo observacional, analítico de caráter longitudinal retrospectivo, baseado na análise de prontuários de pacientes submetidos a três semanas de atendimentos fisioterapêuticos realizados de maneira on-line e em grupo no período de setembro a dezembro de 2020. Resultados: Foram avaliados os prontuários de 25 participantes, considerando como desfecho primário a sensação dolorosa, avaliada pela Escala Numérica da Dor, e como desfechos secundários a incapacidade relacionada à dor lombar, avaliado pelo Roland Morris Disability Questionnaire, e as perspectivas do paciente quanto aos atendimentos, antes e após a intervenção. Após a análise dos dados, observou-se uma redução significativa da dor $(0,007)$ e melhora da incapacidade $(0,002)$ depois das três semanas de intervenção, além de relatos positivos trazidos pelos pacientes com relação à experiência de 
telerreabilitação. Conclusão: A telerreabilitação em grupo mostrou-se eficaz na melhora da dor e incapacidade, mostrando-se satisfatória para os pacientes com dor lombar crônica.

Palavras-chave: Exercício físico; Reabilitação; Autogestão; Fisioterapia.

\begin{abstract}
Objective: To evaluate the effects of group telerehabilitation on pain and disability in patients with chronic low back pain. Methodology: It is a longitudinal retrospective analytical observational study based on the analysis of medical records of patients who underwent three weeks of on-line physical therapy performed in group between september to december 2020. It was evaluated 25 records, considering as the primary outcome the sensation of pain, using Numerical Pain Rating Scale, and as secondary outcomes the disability related to low back pain, evaluated through Roland Morris Disability Questionnaire, and the patient's perspectives regarding care, before and after the intervention. Results: After data analysis, it was observed a significant reduction in pain intensity (0.007) and disability (0.002) after three weeks of intervention, in addition to positive reports from the patients regarding the experience of telerehabilitation. Conclusion: Group telerehabilitation was effective in improving pain and disability, being satisfactory for patients with chronic low back pain.
\end{abstract}

Keywords: Exercise; Rehabilitation; Self-management; Physical therapy.

\title{
Resumen
}

Objetivo: Evaluar los efectos de la telerrehabilitación grupal sobre el dolor y la discapacidad en pacientes con lumbalgia crónica. Metodología: Se trata de estudio longitudinal observacional, analítico, retrospectivo, basado en el análisis de historias clínicas de pacientes que se sometieron a tres semanas de sesiones de fisioterapia realizadas virtualmente y en grupos de septiembre a diciembre de 2020. Resultados: Las historias clínicas de 25 participantes fueron evaluadas, considerando la sensación de dolor como resultado primario, según la escala numérica de dolor, y como resultados secundarios, la discapacidad relacionada con el dolor lumbar, según lo evaluado por el Cuestionario de Discapacidad de Roland Morris, y las perspectivas del paciente con respecto a la atención, antes y después de la intervención. Tras el análisis de los datos, se observó una reducción significativa del dolor $(0,007)$ y una mejora en la discapacidad $(0,002)$ después de las tres semanas de intervención, además de los informes positivos aportados por los pacientes con respecto a la experiencia de telerrehabilitación. Conclusión: La telerrehabilitación grupal demostró ser efectiva para mejorar el dolor y la discapacidad, siendo satisfactoria para los pacientes con lumbalgia crónica.

Palabras clave: Ejercicio físico; Rehabilitación; Autogestión; Fisioterapia.

\section{Introdução}

A dor lombar é uma condição que atinge uma significativa parcela da população brasileira, apresentando uma prevalência de aproximadamente $18,5 \%$, sendo maior no sexo feminino e com idade média de início aos 35 anos (Romero, et al., 2018). Define-se dor lombar como aquela situada entre as últimas costelas e a linha glútea superior, podendo apresentar ou não dor referida no membro inferior. Quando permanece por mais de 12 semanas, é denominada dor lombar crônica (Airaksinen, et al., 2006). Além disso, é considerada a principal causa de incapacidade no mundo, sendo, ainda, um dos fatores que sobrecarregam os sistemas de saúde, especialmente de países de baixa e média renda (Hartvigsen, et al., 2018; Raja, et al., 2020).

O tratamento para essa afecção engloba diversas modalidades, dentre elas, o exercício físico regular é a mais recomendada, assim como medidas educativas baseadas em princípios terapêuticos comportamentais, já que esta combinação gera melhora na função física, capacidade funcional e acelera o retorno ao trabalho. Não há unanimidade sobre qual modalidade de exercícios é a mais eficaz para o tratamento da dor lombar (Yamato, et al., 2015; Chenot, et al., 2017). Desta forma, levando em consideração a sua natureza multifatorial, os atendimentos em grupo aparecem como uma opção considerável e têm se mostrado tão eficazes quanto os atendimentos em formato individual, principalmente nas condições atuais e necessidade de interação e relação interpessoal dessa população (Savigny, et al., 2009).

Mesmo diante da realidade de grupos terapêuticos voltados para pacientes com dor lombar crônica, a alta demanda por tratamento dessa condição ainda é um grande desafio para Sistemas de Saúde. Nesse contexto, Du et al. (2020) apontam que muitos países vêm adotando a prática de promoção à saúde apoiada pelas tecnologias de informação, conhecida como eHealth. Programas de autogerenciamento da dor lombar através de e-Health apresentam altas taxas de adesão, melhora na 
intensidade da dor e incapacidade. A prática apresenta muitos benefícios reconhecidos, tais como a acessibilidade e economia que o usuário faz para ter acesso às informações em saúde; além de permitir a interação dos profissionais de saúde com os pacientes, rastreando as mudanças na sua doença ao longo do tempo e fornecendo orientações em tempo real. Além disso, de acordo com Dias et al. (2021), as tecnologias de informação parecem ser ferramentas favoráveis para o tratamento de disfunções cuja prática de exercício físico é recomendada. Essa modalidade já estava em crescimento e, com o início da pandemia do novo coronavírus no fim de 2019, ganhou maior visibilidade na área da fisioterapia no Brasil com a regulamentação dos serviços de teleconsulta e telemonitoramento por meio da resolução n ${ }^{\circ} 516$ do Conselho Federal de Fisioterapia e Terapia Ocupacional (COFFITO), publicada em 23 de março de 2020 (Slattery, et al., 2017; COFFITO, 2020).

A realização de programas de telerreabilitação envolvendo terapias conservadoras, tais como exercícios físicos, pode promover melhora significativa na dor e incapacidade e ainda, aumentar o engajamento no tratamento, melhorar a compreensão da dor e diminuir as intenções de cirurgias por parte dos participantes com dor lombar crônica (Shebib, et al., 2019). No entanto, conforme pontua Nicholl et al. (2017), a literatura ainda apresenta-se heterogênea e inconclusiva quanto à eficácia de programas de cuidados realizados por meio digital e em grupo para o autogerenciamento da dor lombar crônica.

Diante deste cenário, este estudo tem como objetivo avaliar os efeitos da telerreabilitação em grupo com duração de 3 semanas, em pacientes com dor lombar crônica.

\section{Metodologia}

\section{Características do estudo e participantes:}

Trata-se de um estudo observacional analítico de caráter longitudinal retrospectivo. A amostra foi composta por prontuários on-line de pacientes com dor lombar crônica atendidos em componente curricular prático remoto de uma universidade brasileira. Os dados foram referentes às avaliações e intervenções de atendimentos realizados de maneira virtual, por meio de videochamadas pela plataforma Google Meet e pelo aplicativo de mensagens WhatsApp e registrados no Google Drive.

Os pacientes foram recrutados por conveniência através de divulgação em redes sociais (WhatsApp e Instagram). Foram incluídos no estudo todos os prontuários coletados durante os atendimentos on-line no período de setembro a dezembro de 2020. Aqueles com dados incompletos ou em que o paciente faltou mais de 3 dias de atendimento, foram excluídos da análise.

Os pacientes atendidos possuíam dor lombar crônica e idade superior a 18 anos e não poderiam apresentar dor lombar específica, indisponibilidade de horário para os atendimentos síncronos e mau risco de prognóstico verificado por meio do questionário de triagem Start Back Screening Tool (pontuação total > que 3, e na subescala psicossocial > 3 pontos) (Fritz, et al., 2011; Hill, et al., 2011).

O presente estudo foi aprovado pelo Comitê de Ética em Pesquisa (CEP) da faculdade local, sob o parecer de $n^{\circ}$ : 4.545.860. Para tanto, foi respeitada a autonomia e a garantia do anonimato dos participantes, assegurando sua privacidade quanto a dados confidenciais, como rege a resolução 466/12 do Conselho Nacional de Saúde e a declaração de Helsinki para pesquisa com seres humanos. Logo após aprovação do referido comitê, todos os voluntários foram contatados novamente para autorizarem, de forma voluntária, a inclusão dos seus dados armazenados em prontuário no estudo, assinando o Termo de Consentimento Livre e Esclarecido (TCLE), o qual foi enviado por e-mail.

\section{Procedimentos para coleta/extração de dados do prontuário:}

Após assinatura do TCLE, os dados foram extraídos do banco de prontuários virtual armazenados no Google Drive e 
em seguida realizada a sua análise. Um único pesquisador, previamente treinado, foi responsável pela extração, respeitando os princípios para tratamento de dados pessoais, conforme recomenda a Lei Geral de Proteção de Dados Pessoais (LGPD 13709/2018). Para preservar o anonimato das informações colhidas, os dados foram catalogados em uma planilha de excel, utilizando códigos. Após garantido o anonimato das informações, a planilha foi transmitida para um segundo pesquisador para fins de análise estatística e descritiva dos dados.

\section{Procedimentos de aquisição dos dados:}

Os dados que foram extraídos dos prontuários foram coletados durante atendimentos on-line realizados por meio de videochamadas pela plataforma Google Meet e pelo aplicativo de mensagens WhatsApp.

Para os atendimentos, inicialmente foi realizada uma triagem dos participantes por meio do instrumento Start Back Screening Tool, visando classificar o risco de mau prognóstico de pacientes com dor lombar e/ou lombociatalgia em subgrupos: de baixo, médio e alto risco (Fritz, et al., 2011; Hill, et al., 2011).

Após a triagem, os participantes foram avaliados utilizando uma ficha de avaliação que continha informações pessoais (nome, endereço, naturalidade, telefone para contato, sexo e ocupação principal), dados clínicos (diagnóstico clínico, uso de medicação, comorbidades, história de queda ou acidentes nos últimos meses com suspeita de fratura, intensidade da dor lombar e queixa principal) e questões relacionadas à sua expectativa quanto ao programa de telerreabilitação.

Em seguida, foram avaliadas a sensação dolorosa e a incapacidade relacionada à dor lombar, utilizando os seguintes instrumentos:

Escala numérica da dor: utilizada para mensuração da sensação dolorosa, esta escala apresenta valores de zero a dez, onde 0 (zero) corresponde a ausência de dor e 10 (dez) diz respeito à pior dor imaginável e/ou experienciada pelo paciente (Scott \& Huskisson, 1976). Assim, quanto maior for número indicado, proporcionalmente maior será a dor lombar referida naquele instante.

Roland Morris Disability Questionnaire: É um questionário desenvolvido por Roland e Morris (Roland \& Morris, 1983), e teve sua tradução, adaptação e validação para o Brasil realizada por Nusbaum, et al. (2001). Foi utilizado antes e após a intervenção para medir a incapacidade associada à dor lombar. Ele é constituído por 24 perguntas que descrevem atividades cotidianas que são difíceis de realizar devido à dor lombar e devem ser respondidas com "sim" ou "não". O resultado pode variar de 0 a 24, onde quanto maior for o número de respostas "sim", maior será a incapacidade.

Após a avaliação, os participantes iniciaram um protocolo de intervenção, de 3 semanas, composto por momentos síncronos (realizados duas vezes por semana) e assíncronos (realizados quatro vezes por semana). As sessões síncronas foram compostas por exercícios que consistiram de 5 a 10 minutos iniciais de aquecimento, 40 minutos de exercícios voltados para a coluna com base no Método Pilates e de 5 a 10 minutos finais de relaxamento, totalizando 60 minutos por sessão e 120 minutos por semana. Já os procedimentos durante os momentos assíncronos envolveram orientações direcionadas ao autocuidado, autogerenciamento, ênfase no bem estar e melhora dos hábitos de vida. O acompanhamento de tais atividades foi realizado através de um grupo no Whatsapp, onde os participantes ficavam livres para interagir e expressar suas expectativas, metas e realização das atividades propostas.

Todas as atividades síncronas foram realizadas em grupo e supervisionadas por um docente e dois monitores devidamente treinados e capacitados. O protocolo de intervenção foi realizado por meio de exercícios on-line, de maneira síncrona, na qual foi necessário o encontro virtual e on-line dos participantes e dos terapeutas, além de orientações sobre cuidados e autogerenciamento da dor, assincronamente.

Os dados referentes à sensação dolorosa, incapacidade relacionada à dor lombar e as questões sobre a expectativa do paciente quanto à telerreabilitação foram avaliados no momento pré (T1) e pós intervenção (T2). 


\section{Análise Estatística:}

A análise estatística foi realizada por meio do Software Statistical Package for the Social Sciences (SPSS) versão 20.0 para Windows. Na estatística descritiva utilizou-se média e desvio padrão como medidas de tendência central e de dispersão, respectivamente, para as variáveis quantitativas, e frequência e porcentagem para as variáveis categóricas. Para verificar a normalidade dos dados foi utilizado o teste de Shapiro-Wilk, constatando que os dados apresentaram-se normais. Para analisar a comparação dos desfechos (sensação dolorosa e incapacidade relacionada à dor lombar) nos tempos pré e pós intervenção foi utilizado o teste t pareado. Foi realizada uma análise por intenção de tratar, levando em consideração o método da última observação realizada. O nível de significância utilizado foi de $5 \%(p<0,05)$.

\section{Resultados}

Um total de 25 indivíduos foram selecionados para participarem do estudo. Destes, 9 não finalizaram os atendimentos, como demonstrado no fluxograma abaixo (Figura 1).

Figura 1: Fluxograma do estudo.

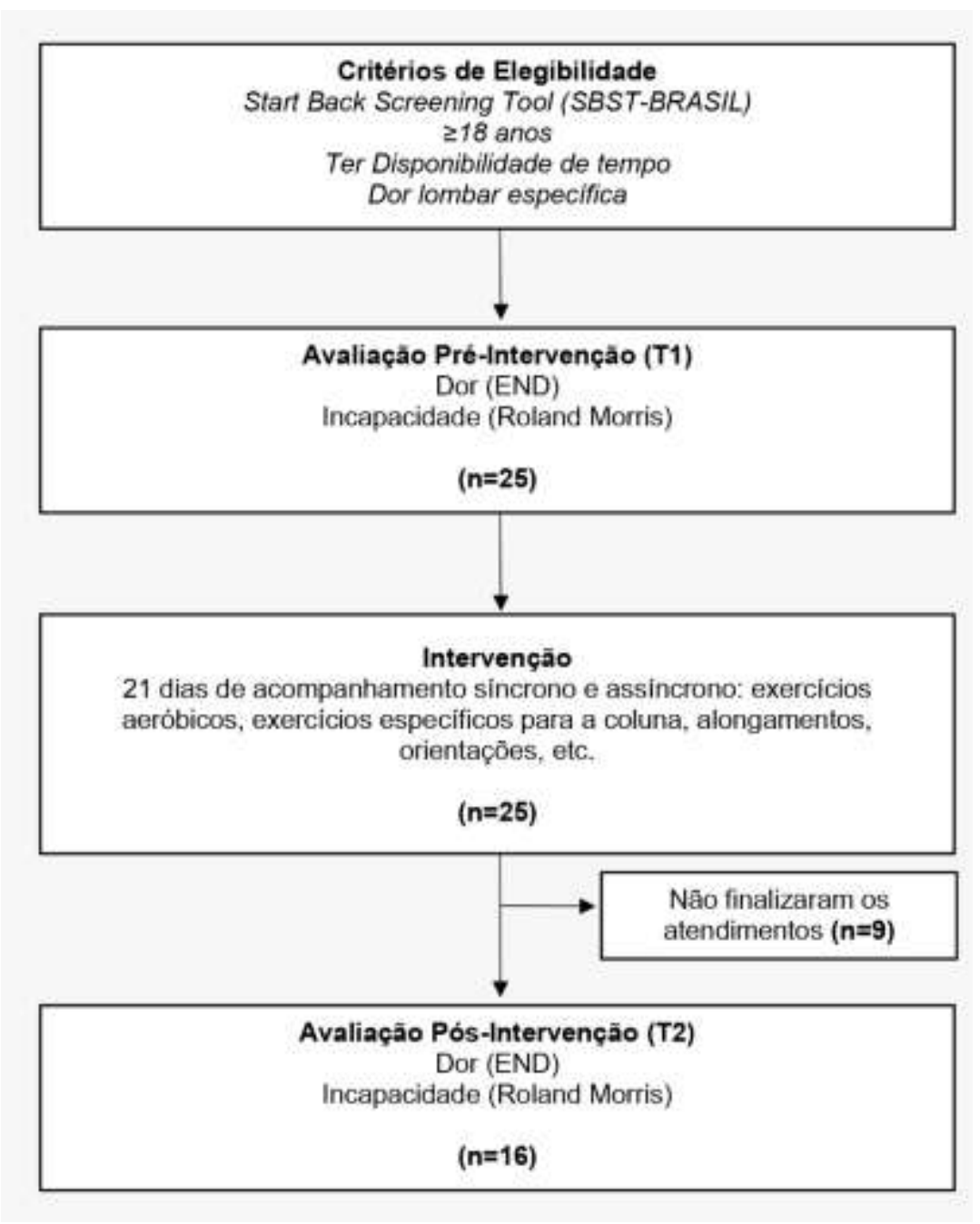

Fonte: Autores.

Os resultados mostraram uma redução estatisticamente significativa $(\mathrm{p}<0,05)$ na dor, bem como para incapacidade, como apresentado abaixo na Tabela 1. 
Tabela 1: Média e desvio padrão (DP) das variáveis dor e incapacidade nos dois tempos de avaliação.

\begin{tabular}{ccccc}
\hline Variáveis & T1 & T2 & T1 x T2 & $\mathrm{p}^{*}$ \\
Dódia (DP) & Média (DP) & (IC 95\%) & \\
& $3,8(2,6)$ & $2,4(1,5)$ & $0,41-2,31$ & $0,007^{*}$ \\
Incapacidade (Roland Morris) & $6,0(3,7)$ & $4,5(3,7)$ & $0,25-2,78$ & $0,002^{*}$ \\
\hline
\end{tabular}

Desvio Padrão (DP); Intervalo de Confiança (IC); Antes intervenção (T1); Após a intervenção (T2); END: Escala Visual Numérica. ${ }^{*} \mathrm{p}<0,05$.

Fonte: Autores.

Conforme demonstrado nos resultados, a dor apresentou uma queda de 1,4 pontos na escala numérica da dor. Com relação a incapacidade, foi notada uma redução 1,5 pontos através dos escores do questionário Roland Morris.

A Tabela 2 apresenta dados referentes às expectativas dos participantes quanto ao programa de exercícios e ao uso de tecnologia, antes do protocolo de intervenção. Na tabela 3 podemos observar suas perspectivas após realizarem o protocolo de intervenção.

Tabela 2: Expectativas dos participantes referentes ao momento pré intervenção.

\begin{tabular}{|c|c|c|}
\hline $\begin{array}{c}\text { Quais são suas expectativas para o programa } \\
\text { de atividades diárias? } \\
\end{array}$ & $\mathrm{n}(\%)$ & Exemplo de fala dos participantes \\
\hline $\begin{array}{l}\text { Positiva } \\
\text { Negativa }\end{array}$ & $\begin{array}{l}25(100 \%) \\
0(0 \%)\end{array}$ & $\begin{array}{c}\text { "Ajudar ao retorno das atividades físicas em } \\
\text { casa, alívio das dores, aprender novos } \\
\text { exercícios de relaxamento para alívio das } \\
\text { dores". }\end{array}$ \\
\hline $\begin{array}{c}\text { Você acha que a utilização de tecnologias será } \\
\text { uma barreira que irá dificultar sua adesão ao } \\
\text { programa? }\end{array}$ & $\mathrm{n}(\%)$ & Exemplo de fala dos participantes \\
\hline Não & $22(88 \%)$ & $\begin{array}{l}\text { "Não, porque acredito que os terapeutas } \\
\text { estejam bem orientados para realizarem as } \\
\text { atividades. As atividades não necessitam de } \\
\text { nenhum equipamento específico e são } \\
\text { exercícios que tenho interesse de praticar". } \\
\text { "É um desafio por não saber manusear algumas } \\
\text { ferramentas, mas possuo o auxílio dos } \\
\text { familiares para ajudar nestes momentos". }\end{array}$ \\
\hline
\end{tabular}

Fonte: Autores. 
Tabela 3: Perspectiva dos participante referentes ao momento pós intervenção.

\begin{tabular}{|c|c|c|}
\hline $\begin{array}{c}\text { Para você, o programa de atividades diárias foi } \\
\text { melhor ou pior do que esperava? }\end{array}$ & $\mathbf{n}(\%)$ & Exemplo de fala dos participantes \\
\hline $\begin{array}{l}\text { Melhor } \\
\text { Pior }\end{array}$ & $\begin{array}{c}16(100 \%) \\
0(0 \%)\end{array}$ & $\begin{array}{c}\text { "Bem melhor, superou todas as minhas } \\
\text { expectativas". }\end{array}$ \\
\hline $\begin{array}{l}\text { Você acha que a utilização de tecnologias foi uma } \\
\text { barreira ou um facilitador para a sua adesão ao } \\
\text { programa? }\end{array}$ & $\mathbf{n}(\%)$ & Exemplo de fala dos participantes \\
\hline Barreira & $0(0 \%)$ & \\
\hline Facilitador & $13(81 \%)$ & $\begin{array}{c}\text { "Facilitador, talvez eu nem fosse se fosse } \\
\text { presencial". }\end{array}$ \\
\hline Ambos & $3(18 \%)$ & $\begin{array}{l}\text { "Existiu ambos os momentos. A instabilidade na } \\
\text { conexão, a busca por um posicionamento e } \\
\text { ambiente adequado para desempenhar as } \\
\text { atividades, além de uma dificuldade na } \\
\text { compreensão dos comandos para corrigir as } \\
\text { posturas inadequadas na realização dos } \\
\text { exercícios, foram as principais barreiras } \\
\text { elencadas. Como facilitador, a oportunidade de } \\
\text { poder participar do atendimento no contexto em } \\
\text { que estamos vivenciando, e assim poder } \\
\text { proporcionar uma melhora da saúde e qualidade } \\
\text { de vida". }\end{array}$ \\
\hline
\end{tabular}

Fonte: Autores.

\section{Discussão}

Conforme apresentado nos resultados deste estudo, após a telerreabilitação realizada em grupo para pacientes com dor lombar crônica, podemos observar uma melhora nos desfechos dor e incapacidade, avaliados por meio da Escala Numérica da Dor e do Questionário Roland Morris, respectivamente, após três semanas de tratamento, composto por momentos síncronos e assíncronos que envolviam exercícios, orientações, autogerenciamento e autocuidado.

A dor crônica, caracterizada por sintomas presentes por três meses ou mais (Taub, et al., 1998), promove alterações na função física que, consequentemente, contribuem negativamente para a qualidade de vida dos indivíduos. Além disso, aspectos psicossociais influenciam na multifatoriedade da dor lombar, repercutindo na experiência dolorosa e incapacidade (Hartvigsen, 2018). Geneen et al. (2017) e Chou et al. (2017) demonstram que a atividade física e o exercício são benéficos para a melhora da dor. Similarmente, Vanti et al. (2019) sugeriram que as práticas dessas intervenções atuam positivamente na melhora da dor e incapacidade dessa população.

Por sua vez, segundo Owen et al. (2020), em sua revisão sistemática, diversas modalidades de exercícios demonstraram ser eficazes na melhora da função física, saúde mental, força e resistência muscular, além da redução da dor em pacientes com dor lombar crônica não específica. Dentre as modalidades avaliadas pelos autores, o Pilates, exercícios resistidos, de estabilização e exercícios aeróbicos, influenciaram na redução da dor nestes pacientes. Corroborando com esses achados, o protocolo de intervenção aplicado pelo presente estudo abrangeu uma variedade de atividades, contemplando exercícios baseados nos fundamentos do método Pilates, aeróbicos e ativos-livres, estruturados de forma gradual e adaptado para cada indivíduo. Além disso, nos momentos assíncronos, como proposta de ações continuadas abrangendo os aspectos biopsicossociais da dor lombar crônica, foram incluídas atividades como meditação, auto alongamento, dança, caminhada, atividades artesanais, lúdicas e que proporcionavam bem-estar e interação social.

Nossa proposta tinha o objetivo de perpetuar ações além do protocolo de exercício, inserindo-as nos hábitos de vida dos indivíduos. Desta forma, diferentes opções de atividades eram sugeridas aos pacientes, para que, de acordo com suas 
preferências, o autogerenciamento da sua condição de saúde fosse facilitado. Segundo Barlow et al. (2002), o autogerenciamento é uma ferramenta que visa incentivar a administração e empoderar o paciente para torná-lo protagonista do seu cuidado em saúde, como também colaborar com uma boa relação entre paciente e profissional de saúde. Du et al. (2017), em sua revisão sistemática para verificar a eficácia dos programas de autogestão na dor lombar crônica, mostra que o programa de autocuidado tem efeito moderado na intensidade da dor e efeitos pequenos a moderados na incapacidade, corroborando com os achados do presente estudo, que encontrou resultados significativos para dor e incapacidade, apesar destes não alcançaram os valores clinicamente importantes descritos pela literatura (Ostelo, et al., 2008).

Uma forma inovadora e que vem cada vez mais sendo aliada ao autogerenciamento é a e- Health. Segundo Du et al. (2020), a prática de saúde apoiada pelas tecnologias de informação demonstra benefícios na melhora da dor e da incapacidade, a curto prazo, em pacientes com dor lombar crônica. A telerreabilitação foi a alternativa proposta para continuidade das condutas no contexto da pandemia da COVID-19, sendo sugerida por Seron et al. (2021) como uma terapia eficaz, apresentando resultados semelhantes à forma presencial e melhor do que não realizar reabilitação para condições como a dor lombar. Dias et al. (2021) concluem que o exercício físico na telerreabilitação pode ser uma opção relevante para o tratamento da dor, função física e qualidade de vida em adultos com deficiência. Similarmente, Cottrell et al. (2017) discutem que a prática remota da reabilitação pode ter sua eficácia comparada aos métodos convencionais de assistência à saúde na melhora da dor e função física em uma gama de condições musculoesqueléticas. O estudo de Peterson (2018) concluiu que a telerreabilitação, como ferramenta de acompanhamento longitudinal ao atendimento presencial, provou ser benéfica para pacientes que sofrem de dor lombar, promovendo e mantendo uma redução dos níveis de dor.

Com base na perspectiva do paciente, a telerreabilitação também parece ser uma excelente aliada no tratamento de suas condições. De acordo com os achados do presente estudo, os pacientes tratados de forma on-line, além de melhorarem dor e incapacidade, apresentaram expectativas positivas com o tratamento, reportando, em sua maioria, o uso de tecnologias como um facilitador e demonstrando satisfação com os atendimentos. Em seu estudo, Cottrell et al. (2018) encontraram percepções positivas dos pacientes em relação ao uso de telessaúde para tratar condições musculoesqueléticas crônicas. Suas preferências a favor da telessaúde estão relacionadas a redução dos custos e tempo de viagem associados aos atendimentos presenciais, além da flexibilidade de horário para as pessoas que trabalham em horário comercial.

\section{Conclusão}

A telerreabilitação em grupo mostrou-se eficaz na melhora da dor e incapacidade, além de apresentar-se como uma opção positiva pontuada pelos pacientes, evidenciando a importância de uma abordagem multidimensional para indivíduos com dor lombar crônica. Além disso, acreditamos que a telerreabilitação mostrou-se um instrumento de autogerenciamento importante que proporciona uma maior participação dos indivíduos, além de ultrapassar barreiras, tornando o atendimento fisioterapêutico mais acessível.

Potenciais limitações do presente estudo podem ser citadas, como o seu desenho não ser experimental, não permitindo investigar a causalidade dos resultados. Além disso, o pequeno número de sujeitos e o curto tempo de intervenção e investigação, não possuindo follow-up, também podem ser pontuados. Desta forma, sugerimos que futuros estudos sejam realizados também objetivando verificar os efeitos da telerreabilitação em indivíduos com dor lombar, entretanto, com um desenho experimental, incluindo um maior número de participantes, com um tempo prolongado de intervenção e acompanhamento. 


\section{Agradecimentos}

Agradecemos à turma que cursou o $7^{\circ}$ período do curso de fisioterapia da FACISA/UFRN pela participação nos atendimentos realizados.

\section{Referências}

Airaksinen, O., et al. (2006). Chapter 4 European guidelines for the management of chronic nonspecific low back pain. Eur spine J. 15(Suppl 2), 192-300.

Barlow, J., Wright, C., Sheasby, J., Turner, A., \& Hainsworth, J. (2002). Self-management approaches for people with chronic conditions: a review. Patient Educ Couns. 48(2), 177-87.

Chenot, J-F., Greitemann, B., Kladny, B., Petzke, F., Pfingsten, M., \& Schorr, S. G. (2017). Non-Specific Low Back Pain. Dtsch Arztebl Int. 114(51-52), 88390 .

Chou, R, , et al. (2017). Nonpharmacologic Therapies for Low Back Pain: A Systematic Review for an American College of Physicians Clinical Practice Guideline. Ann Intern Med. 166(7), 493-505.

COFFITO. Resolução $n^{o} 516$ de 20 de Março de 2020. Teleconsulta, Telemonitoramento e Teleconsultoria. Diário Oficial da União. https://www.coffito.gov.br/nsite/?p=15825

Cottrell, M. A., et al. (2017). Real-time telerehabilitation for the treatment of musculoskeletal conditions is effective and comparable to standard practice: a systematic review and meta-analysis. Clin Rehabil. 31(5), 625-38.

Cottrell, M. A., et al. (2018). Patients are willing to use telehealth for the multidisciplinary management of chronic musculoskeletal conditions: A crosssectional survey. J Telemed Telecare. 24(7), 445-52.

Dias, J. F., et al. (2021). Effectiveness of exercises by telerehabilitation on pain, physical function and quality of life in people with physical disabilities: a systematic review of randomised controlled trials with GRADE recommendations. Br J Sports Med. 55(3), 155-62.

Du, S., et al. (2017). Self-management program for chronic low back pain: A systematic review and meta-analysis. Patient Educ Couns. 100(1), 37-49.

Du, S., Liu, W., Cai, S., Hu, Y., \& Dong, J. (2020). The efficacy of e-health in the self-management of chronic low back pain: A meta analysis. Int J Nurs Stud. 106(103507), 01-12.

Fritz, J. M., Beneciuk, J. M., \& George, S. Z. (2011). Relationship between categorization with the STarT Back Screening Tool and prognosis for people receiving physical therapy for low back pain. Phys Ther. 91(5), 722-32.

Geneen, L J., Moore, R. A., Clarke, C., Martin, D., Colvin, L. A., \& Smith, B. H. (2017). Physical activity and exercise for chronic pain in adults: an overview of Cochrane Reviews. Cochrane database Syst Rev. (4), 01-75.

Hartvigsen, J., Hancock, M. J., Kongsted, A., Louw, Q., Ferreira, M. L., Genevay, S., et al. (2018). What low back pain is and why we need to pay attention. Lancet. 391(10137), 2356-67.

Hill, J. C., et al. (2011). Comparison of stratified primary care management for low back pain with current best practice (STarT Back): a randomised controlled trial. Lancet. 378(9802), 1560-71.

Nicholl, B. I., et al. (2017). Digital Support Interventions for the Self-Management of Low Back Pain: A Systematic Review. J Med Internet Res. 19(5), 01-21.

Nusbaum, L., Natour, J, Ferraz, M. B., \& Goldenberg, J. (2001). Translation, adaptation and validation of the Roland-Morris questionnaire-Brazil RolandMorris. Brazilian J Med Biol Res. 34(2), 203-10.

Ostelo, R. W. J. G.,. et al. (2008). Interpreting change scores for pain and functional status in low back pain: towards international consensus regarding minimal important change. Spine. 33(1), 90-4.

Owen, P. J., Miller, C. T., Mundell, N. L., Verswijveren, S. J. J. M., Tagliaferri, S. D., Brisby, H., et al. (2020). Which specific modes of exercise training are most effective for treating low back pain? Network meta-analysis. Br J Sports Med. 54(21), 1279-87.

Peterson, S. (2018). Telerehabilitation booster sessions and remote patient monitoring in the management of chronic low back pain: A case series. Physiother Theory Pract. 34(5), 393-402.

Raja, S. N., et al. (2020). The revised International Association for the Study of Pain definition of pain: concepts, challenges, and compromises. Pain. 161(9), 1976-82.

Roland, M., \& Morris, R. (1983). A study of the natural history of back pain. Part I: development of a reliable and sensitive measure of disability in low-back pain. Spine. 8(2), 141-4.

Romero, D. E., Santana, D., Borges, P., Marques, A., Castanheira, D., Rodrigues, J. M., et al. (2018). Prevalência, fatores associados e limitações relacionados ao problema crônico de coluna entre adultos e idosos no Brasil. Cad Saude Publica. 34(2), 01-15.

Savigny, P., Watson, P., \& Underwood, M. Early management of persistent non-specific low back pain: summary of NICE guidance. (2009). Br J Sports Med. 338(b1805), 1441-45 
Research, Society and Development, v. 10, n. 9, e6710917741, 2021

(CC BY 4.0) | ISSN 2525-3409 | DOI: http://dx.doi.org/10.33448/rsd-v10i9.17741

Scott, J., \& Huskisson, E. C. (1976). Graphic representation of pain. Pain. 2(2), 175-84.

Seron, P., Oliveros, M. J., Gutierrez-Arias, R., Fuentes-Aspe, R., Torres-Castro, R., Merino-Osorio, C., et al. (2021). Effectiveness of telerehabilitation in physical therapy: A rapid overview. Phys Ther. 101(6), 01-52.

Shebib, R., Bailey, J. F., Smittenaar, P., Perez, D. A., Mecklenburg, G., \& Hunter, S. (2019). Randomized controlled trial of a 12-week digital care program in improving low back pain. NPJ Digit Med. 2(1), 02-8.

Slattery, B. W., et al. (2017). Protocol for a systematic review with network meta-analysis of the modalities used to deliver eHealth interventions for chronic pain. Syst Rev. 6(45), 01-8.

Taub, N. S., Worsowicz, G. M., Gnatz, S. M., \& Cifu, D. X. (1998). 1. Definitions and diagnosis of pain. Arch Phys Med Rehabil. 79(3), 49-53.

Vanti, C., Andreatta, S., Borghi, S., Guccione, A. A., Pillastrini, P., \& Bertozzi, L. (2019). The effectiveness of walking versus exercise on pain and function in chronic low back pain: a systematic review and meta-analysis of randomized trials. Disabil Rehabil. 41(6), 622-32.

Yamato, T. P., et al. (2015). Pilates for low back pain. Cochrane database Syst Rev. 1(7), 01-62. 